\title{
ON THE VOLTERRA DELAY-INTEGRO-DIFFERENTIAL EQUATION WITH LAYER BEHAVIOR AND ITS NUMERICAL SOLUTION
}

\author{
GABIL M. AMIRALIYEV AND ÖMER YAPMAN
}

Received 12 October, 2017

\begin{abstract}
In this paper, we analyze the convergence of the fitted mesh method applied to singularly perturbed Volterra delay-integro-differential equation. Our mesh comprises a special nonuniform mesh on the first subinterval and uniform mesh on another part. Error estimates are obtained using difference analogue of Gronwall's inequality with delay. A numerical test that confirms the theoretical results is presented.
\end{abstract}

2010 Mathematics Subject Classification: 65L11; 65L12; 65L20; 65R20

Keywords: Volterra delay-integro-differential equation, singular perturbation, finite difference, uniform convergence

\section{INTRODUCTION}

Volterra delay-integro-differential equations (VDIDEs) have a major influence on the field of science such as ecology, medicine, physics, biology and so on [4, 6, 14]. These equations play a significant role in modelling of some phenomena in engineering and sciences, and hence have led researchers to develop a theory and numerical computation and analysis for VDIDEs.

Here we shall concerned with the development of fitted difference method for singularly perturbed Volterra delay-integro-differential equation (SPVDIDE):

$$
L u:=\varepsilon u^{\prime}+a(t) u+\int_{t-r}^{t} K(t, s) u(s) d s=f(t), t \in I,
$$

subject to

$$
u(t)=\varphi(t), t \in I_{0},
$$

where $I=(0, T]=\bigcup_{p=1}^{m} I_{p}, I_{p}=\left\{t: r_{p-1}<t \leq r_{p}\right\}, 1 \leq p \leq m$ and $r_{s}=s r$, for $0 \leq s \leq m, \bar{I}=[0, T]$ and $I_{0}=[-r, 0] . \varepsilon \in(0,1]$ is the perturbation parameter and $r$ is a constant delay, which is independent of $\varepsilon . a(t) \geq \alpha>0, f(t)(t \in \bar{I}), \varphi(t)$ 
$\left(t \in I_{0}\right)$ and $K(t, s)((t, s) \in \bar{I} \times \bar{I})$ are assumed to be sufficiently smooth functions such that the solution, $u(t)$, has initial layer at $t=0$ for small values of $\varepsilon$.

Singularly perturbed differential equations are typically characterized by a small parameter $\varepsilon$ multiplying some or all of the highest order terms in the differential equation. In general, the solutions of such equations exhibit multiscale phenomena. Within certain thin subregions of the domain, the scale of some derivatives is significantly larger than other derivatives. These thin regions of rapid change are called, boundary or interior layers, as appropriate. Such type of equations occur frequently in mathematical problems in the sciences and engineering for example, in fluid flow at high Reynold number, electrical networks, chemical reactions, control theory, the equations governing flow in porous media, the drift-diffusion equations of semi-conductor device physics, and other physical models [5, 16, 17]. It is wellknown that standard discretization methods do not work well for these problems as they often produce oscillatory solutions which are inaccurate if the perturbed parameter $\varepsilon$ is small. To obtain robust numerical methods it is necessary to fit the coefficients (fitted operator methods) or the mesh (fitted mesh methods) to the behavior of the exact solution $[1,2,5,10,15,19,22]$ (see also references cited in them). For a survey of early results in the theoretical analysis of singularly perturbed Volterra integro-differential equations (VIDEs) and in the numerical analysis and implementation of various techniques for these problems we refer to the book [11]. An analysis of approximate methods when applied to singularly perturbed VIDEs can also be found in $[12,18,20]$.

In the last few years, a considerable amount of effort has been devoted to the numerical solution of VDIDEs. An overview of the approximate methods for VDIDEs may be obtained from $[3,7,9,21,24,25]$.

The above mentioned papers, related to VDIDEs were only concerned with the regular cases, i.e., in the absence of initial/boundary layers. SPVDIDEs also frequently arise in many scientific applications. Wu and Gan [23] investigated error behaviour of linear multistep methods applied to SPVDIDEs and derived global error estimates $A(\alpha)$-stable linear multistep methods with convergent quadrature rule. $\mathrm{He}$ and $\mathrm{Xu}$ [8] discussed the exponential stability of impulsive SPVDIDEs. Amiraliyev and Yilmaz [2] gave an exponentially fitted difference method on a uniform mesh for (1.1)-(1.2) except for a delay term in differential part and shown that the method is first-order convergent uniformly in $\varepsilon$. A useful discussion of uniform convergence on a fitted mesh, for another form of SPVDIDEs have been investigated in [13].

In this paper, the main goal is to give a finite difference scheme on a special piecewise uniform mesh on the first subinterval and uniform mesh on the rest of the interval for the numerical solution of the initial-value problem (1.1)-(1.2). The construction of the difference scheme is based on the method of integral identities by using appropriate interpolating quadrature rules with remainder term in integral form. In Section 2 , we construct the finite difference discretization and introduce a special piecewise 
uniform mesh. In Section 3, the error analysis for the approximate solution is presented and the method is shown theoretically to be uniformly (with respect to the singular perturbation parameter) convergent. Numerical results are given in Section 4 which validate that in practice the method is first order uniformly accurate. The paper ends with a summary of the main conclusions.

Notation: Throughout the paper we use $C$ to denote a generic positive constant independent of the perturbation parameter and mesh.

Assumption: We also will assume that $\varepsilon \leq C N^{-1}$, as is generally the case in practise.

\section{THE MESH AND DIFFERENCE SCHEME}

In this section, a special piecewise uniform mesh on $[0, T]$ is introduced and the difference scheme consisting of upwind type scheme for differential part and a composite rectangle integration of explicit type for integral part is presented for discretizing (1.1)-(1.2).

Before presenting the numerical method for the solution of (1.1)-(1.2) we need the asymptotic estimates for its differential solution.

Lemma 1. For $a, f \in C^{1}[0, T]$,

$$
\left|\frac{\partial}{\partial t} K(t, s)\right| \leq M_{0}<\infty,
$$

the solution of (1.1)-(1.2) satisfies

$$
\begin{gathered}
\|u\|_{\infty} \leq C_{0}, \\
\left|u^{\prime}(t)\right| \leq C\left\{1+\frac{1}{\varepsilon} e^{-\frac{\alpha t}{\varepsilon}}\right\}, 0 \leq t \leq T,
\end{gathered}
$$

where

$$
\begin{gathered}
C_{0}=\left(|\varphi(0)|+\alpha^{-1} \bar{K}\|\varphi\|_{1,0}+\alpha^{-1}\|f\|_{\infty}\right) e^{-\alpha^{-1} \bar{K} T}, \\
\bar{K}=\max _{\bar{I} \times \bar{I}}|K(t, s)|, \\
\|\varphi\|_{1,0}=\int_{-r}^{0}|\varphi(t)| d t .
\end{gathered}
$$

The proof is being established in the analogous way as in $[2,13]$.

Our nonuniform mesh

$$
\bar{\omega}=\left\{0=t_{0}<t_{1}<\ldots<t_{N_{0}-1}<t_{N_{0}}=T ; h_{i}=t_{i}-t_{i-1}\right\},
$$

which consists of $m$ submeshes $\omega_{N, p}(1 \leq p \leq m)$, for an even number $N$, organized as follows 


$$
\begin{gathered}
\omega=\bigcup_{p=1}^{m} \omega_{N, p}, \\
\omega_{N, 1}=\left\{t_{i}=i h^{(1)}, i=1,2, \ldots, N / 2, h^{(1)}=\frac{2 \sigma}{N}, t_{i}=\sigma+\left(i-\frac{N}{2}\right) h^{(2)},\right. \\
\left.i=\frac{N}{2}+1, \ldots, \frac{3 N}{2}, h^{(2)}=\frac{r-\sigma}{N}\right\}, \\
\omega_{N, p}=\left\{t_{i}=r_{p-1}+\left(i-\left(p-\frac{1}{2}\right) N\right) h^{(3)}, i=\left(p-\frac{1}{2}\right) N+1, \ldots,\left(p+\frac{1}{2}\right) N,\right. \\
\left.h^{(3)}=\frac{r}{N}\right\}, \quad 2 \leq p \leq m, r_{s}=s r(0 \leq s \leq m)
\end{gathered}
$$

with transition point $\sigma=\alpha^{-1} \varepsilon \ln N$. Thus the mesh $\omega$ contains $\frac{3 N}{2}$ points on $\omega_{N, 1}$ and $N$ points on each subinterval $\omega_{N, p}(2 \leq p \leq m)$ with total number $N_{0}=(m+$ $\left.\frac{1}{2}\right) N$.

The problem (1.1)-(1.2) is discretized using the relation

$$
h_{i}^{-1} \int_{t_{i-1}}^{t_{i}} L u(t) d t=h_{i}^{-1} \int_{t_{i-1}}^{t_{i}} f(t) d t, \quad 1 \leq i \leq N_{0}
$$

followed by the application appropriate quadrature rules.

Namely, applying right side rectangle rule in (2.1), analogous to [13] we get

$$
h_{i}^{-1} \int_{t_{i-1}}^{t_{i}}\left[\varepsilon u^{\prime}(t)+a(t) u(t)-f(t)\right] d t=\varepsilon u_{\bar{t}, i}+a_{i} u_{i}-f_{i}+R_{i}^{(1)}
$$

with the truncation error

$$
R_{i}^{(1)}=-h_{i}^{-1} \int_{t_{i-1}}^{t_{i}}\left(t-t_{i-1}\right) \frac{d}{d t}[a(t) u(t)-f(t)] d t .
$$

For the remain part in (2.1), after using right side rectangle rule, we have

$$
h_{i}^{-1} \int_{t_{i-1}}^{t_{i}}\left(\int_{t-r}^{t} K(t, s) u(s) d s\right) d t=\int_{t_{i}-r}^{t_{i}} K\left(t_{i}, s\right) u(s) d s+R_{i}^{(2)},
$$

with

$$
R_{i}^{(2)}=-h_{i}^{-1} \int_{t_{i-1}}^{t_{i}}\left(\xi-t_{i-1}\right)\left(\frac{d}{d \xi} \int_{\xi-r}^{\xi} K(\xi, s) u(s) d s\right) d \xi
$$


Also applying the composite left side rectangle rule, we obtain

$$
\int_{t_{i}-r}^{t_{i}} K\left(t_{i}, s\right) u(s) d s=\sum_{j=i-\bar{N}+1}^{i} h_{j} K\left(t_{i}, t_{j-1}\right) u_{j-1}+R_{i}^{(3)},
$$

with

$$
\begin{array}{r}
\bar{N}=\left\{\begin{array}{cc}
\frac{3 N}{2}, & 1 \leq i \leq \frac{3 N}{2} \\
N, & i>\frac{3 N}{2}
\end{array}\right. \\
R_{i}^{(3)}=\sum_{j=i-\bar{N}+1}^{i} \int_{t_{j-1}}^{t_{j}}\left(t_{j}-\xi\right) \frac{d}{d s} K\left(t_{i}, \xi\right) u(\xi) d \xi,
\end{array}
$$

for

$$
1 \leq i \leq \frac{3 N}{2} \text { and } i>\frac{5 N}{2},
$$

and

$$
R_{i}^{(3)}=\sum_{j=i-\bar{N}+1}^{i} \int_{t_{j-1}}^{t_{j}}\left(t_{j}-\xi\right) \frac{d}{d s} K\left(t_{i}, \xi\right) u(\xi) d \xi-\int_{t_{i-N}}^{t_{i}-r} K\left(t_{i}, s\right) u(s) d s,
$$

for

$$
\frac{3 N}{2}<i \leq \frac{5 N}{2} .
$$

Therefore we have the exact equality for the $u(t)$ :

$$
\begin{aligned}
& L_{N} u_{i}:=\varepsilon u_{\bar{t}, i}+a_{i} u_{i}+\sum_{j=i-\bar{N}+1}^{i} h_{j} K\left(t_{i}, t_{j-1}\right) u_{j-1}+R_{i}=f_{i}, 1 \leq i \leq N_{0}, \\
& u_{i}=\varphi_{i},-\frac{3 N}{2} \leq i \leq 0,
\end{aligned}
$$

with remainder term

$$
R_{i}=R_{i}^{(1)}+R_{i}^{(2)}+R_{i}^{(3)}
$$

where $R_{i}^{(k)}(k=1,2,3)$ are given by (2.2), (2.3), (2) and (2.5).

Based on (2.6) we propose the following scheme for approximating (1.1)-(1.2):

$$
\begin{aligned}
& L_{N} y_{i}:=\varepsilon y_{\bar{t}, i}+a_{i} y_{i}+\sum_{j=i-\bar{N}+1}^{i} h_{j} K_{i, j-1} y_{j-1}=f_{i}, 1 \leq i \leq N_{0}, \\
& y_{i}=\varphi_{i},-\frac{3 N}{2} \leq i \leq 0 .
\end{aligned}
$$




\section{ERROR ANALYSIS AND CONVERGENCE}

Let $z_{i}=y_{i}-u_{i}$. Then for the error of the approximate solution $z_{i}$, from (2.6) and (2.7) it follows that

$$
\begin{aligned}
& L_{N} z_{i}=R_{i}, \quad 1 \leq i \leq N_{0}, \\
& z_{i}=0, \quad-\frac{3 N}{2} \leq i \leq 0 .
\end{aligned}
$$

Lemma 2. For the solution of (3.1)-(3.2) holds the estimate

$$
\|z\|_{\infty, \omega} \leq C\|R\|_{\infty, \omega} .
$$

Proof. Once the inequality

$$
\left|\sum_{j=i-\bar{N}+1}^{i} h_{j} K_{i, j-1} z_{j-1}\right| \leq \bar{K} \sum_{j=i-\bar{N}+1}^{i} h_{j}\left|z_{j-1}\right| \leq \bar{K} \sum_{j=1}^{i} h_{j}\left|z_{j-1}\right|,
$$

is satisfied, then by using Lemma 4.1 from [13] we have that

$$
\left|z_{i}\right| \leq \alpha^{-1}\|R\|_{\infty, \omega}+\alpha^{-1} \bar{K} \sum_{j=1}^{i} h_{j}\left|z_{j-1}\right|,
$$

which in turn in view of difference analogue of Gronwall's inequality leads to

$$
\left|z_{i}\right| \leq \alpha^{-1}\|R\|_{\infty, \omega} \exp \left(\alpha^{-1} \bar{K} \sum_{j=1}^{i} h_{j}\right) \leq \alpha^{-1} \exp \left(\alpha^{-1} \bar{K} T\right)\|R\|_{\infty, \omega} .
$$

Thus the proof is completed.

Lemma 3. Under the assumptions of Section 1, the error function $R_{i}$ of the difference scheme satisfies

$$
\|R\|_{\infty, \omega} \leq C N^{-1} \ln N .
$$

Proof. We estimate $R_{i}^{(k)}(k=1,2,3)$ separately. For $R_{i}^{(1)}$, using (2.2) on an arbitrary mesh we have

$$
\left|R_{i}^{(1)}\right| \leq C h_{i}^{-1} \int_{t_{i-1}}^{t_{i}}\left(t-t_{i-1}\right)\left(1+\left|u^{\prime}(t)\right|\right) d t, \quad 1 \leq i \leq N_{0} .
$$

From here by virtue of Lemma 1 we get

$$
\left|R_{i}^{(1)}\right| \leq C\left\{h_{i}+h_{i}^{-1} \varepsilon^{-1} \int_{t_{i-1}}^{t_{i}}\left(t-t_{i-1}\right) e^{-\alpha t / \varepsilon} d t\right\}, 1 \leq i \leq N_{0} .
$$


On the layer region $[0, \sigma]$ this reduces to

$$
\left|R_{i}^{(1)}\right| \leq C h^{(1)}\left(1+\varepsilon^{-1}\right)=C 2 \alpha^{-1} N^{-1} \ln N(\varepsilon+1), \quad 1 \leq i \leq \frac{N}{2},
$$

therefore

$$
\left|R_{i}^{(1)}\right| \leq C N^{-1} \ln N, \quad 1 \leq i \leq \frac{N}{2},
$$

For $i>\frac{N}{2}$, we can write

$$
\begin{aligned}
\left|R_{i}^{(1)}\right| & \leq C\left\{h_{i}+\varepsilon^{-1} \int_{t_{i-1}}^{t_{i}} e^{-\alpha t / \varepsilon} d t\right\}=C\left\{h_{i}+\alpha^{-1}\left(e^{-\alpha t_{i-1} / \varepsilon}-e^{-\alpha t_{i} / \varepsilon}\right)\right\} \\
& =C\left\{h_{i}+\alpha^{-1} e^{-\alpha t_{i-1} / \varepsilon}\left(1-e^{-\alpha h_{i} / \varepsilon}\right)\right\} \leq C\left(h_{i}+\alpha^{-1} e^{-\alpha t_{i-1} / \varepsilon}\right) \\
& \leq C\left(h_{i}+\alpha^{-1} e^{-\alpha \sigma / \varepsilon}\right)=C\left(h_{i}+\alpha^{-1} N^{-1}\right), \frac{N}{2}+1 \leq i \leq N_{0}
\end{aligned}
$$

with

Thereby

$$
h_{i}=h^{(2)}, \frac{N}{2}+1 \leq i \leq \frac{3 N}{2} ; \quad h_{i}=h^{(3)}, \frac{3 N}{2}+1 \leq i \leq N_{0} .
$$

$$
\left|R_{i}^{(1)}\right| \leq C N^{-1}, \frac{N}{2}+1 \leq i \leq N_{0} .
$$

Next for $R_{i}^{(2)}$, according to (2.3) we have

$$
\left|R_{i}^{(2)}\right| \leq \int_{t_{i-1}}^{t_{i}}\left|\int_{\xi-r}^{\xi} \frac{\partial K(\xi, s)}{\partial \xi} u(s) d s+K(\xi, \xi) u(\xi)-K(\xi, \xi-r) u(\xi-r)\right| d \xi .
$$

Taking into consideration the boundless of $\partial K / \partial \xi, K(t, s)$ and Lemma 1 we arrive at

$$
\left|R_{i}^{(2)}\right| \leq C r h_{i}, \quad 1 \leq i \leq N_{0},
$$

which in turn implies that

$$
\left|R_{i}^{(2)}\right| \leq C N^{-1} \ln N
$$

Now estimate $R_{i}^{(3)}$ for $1 \leq i \leq \frac{3 N}{2}$ and $i>\frac{5 N}{2}$ we get

$$
\left|R_{i}^{(3)}\right| \leq \sum_{j=i-\bar{N}+1}^{i} \int_{t_{j-1}}^{t_{j}}\left(t_{j}-\xi\right)\left|\frac{d}{d \xi} K\left(t_{i}, \xi\right)\right||u(\xi)| d \xi \leq C \sum_{j=i-\bar{N}+1}^{i} h_{j}^{2} .
$$

From here it follows that

$$
\left|R_{i}^{(3)}\right| \leq C \bar{N}\left|h^{(1)}\right|^{2} \leq C N^{-1} \ln N, \quad 1 \leq i \leq \frac{N}{2},
$$




$$
\begin{aligned}
& \left|R_{i}^{(3)}\right| \leq C \bar{N}\left|h^{(2)}\right|^{2} \leq C N^{-1}, \frac{N}{2}+1 \leq i \leq \frac{3 N}{2}, \\
& \left|R_{i}^{(3)}\right| \leq C \bar{N}\left|h^{(3)}\right|^{2} \leq C N^{-1}, i>\frac{5 N}{2} .
\end{aligned}
$$

Finally, we estimate $R_{i}^{(3)}$ for $\frac{3 N}{2}<i \leq \frac{5 N}{2}$

$$
\begin{aligned}
\left|R_{i}^{(3)}\right| & \leq \sum_{j=i-N+1}^{i} \int_{t_{j-1}}^{t_{j}}\left(t_{j}-\xi\right)\left|\frac{d}{d \xi} K\left(t_{i}, \xi\right)\right||u(\xi)| d \xi+\left|\int_{t_{i-N}}^{t_{i}-r} K\left(t_{i}, s\right) u(s) d s\right| \\
& \leq C\left(\sum_{j=i-N+1}^{i} h_{j}^{2}+\left|t_{i}-r-t_{i-N}\right|\right) .
\end{aligned}
$$

Since

$$
\begin{aligned}
t_{i}-r-t_{i-N} & =r+\left(i-\frac{3 N}{2}\right) h^{(3)}-r-\sigma-\left(i-\frac{3 N}{2}\right) h^{(2)} \\
& =-\sigma\left[1-\left(i-\frac{3 N}{2}\right) N^{-1}\right]
\end{aligned}
$$

and

$$
\begin{aligned}
& \sum_{j=i-N+1}^{i} h_{j}^{2}=\sum_{j=i-N+1}^{3 N / 2} h_{j}^{2}+\sum_{j=\frac{3 N}{2}+1}^{i} h_{j}^{2}=\left(\frac{3 N}{2}-i+N\right)\left|h^{(2)}\right|^{2}+\left(i-\frac{3 N}{2}\right)\left|h^{(3)}\right|^{2} \\
& \leq N\left(\left|h^{(2)}\right|^{2}+\left|h^{(3)}\right|^{2}\right)=(r-\sigma) h^{(2)}+r h^{(3)}=\left[(r-\sigma)^{2}+r^{2}\right] N^{-1},
\end{aligned}
$$

then

$$
\left|R_{i}^{(3)}\right| \leq C\left(\sigma+N^{-1}\right) \leq C N^{-1} \ln N, \frac{3 N}{2}<i \leq \frac{5 N}{2} .
$$

Now use (3.4)-(3.10) to get the desired inequality (3.3).

Lemma 2 and Lemma 3 give the main result of our paper.

Theorem 1. Let $u$ be solution of (1.1)-(1.2) and $y_{i}$ its approximation by the difference scheme (2.7)-(2.8). Then

$$
\|u-y\|_{\infty, \bar{\omega}} \leq C N^{-1} \ln N .
$$

\section{NUMERICAL RESULTS}

Example 1. First we study the following test problem

$$
\varepsilon u^{\prime}+2 u-\int_{t-1}^{t} u(s) d s=-1+t-\frac{\varepsilon}{2}\left(1-e^{-\frac{2 t}{\varepsilon}}\right), t \in(0,2],
$$




$$
u(t)=1,-1 \leq t \leq 0 .
$$

The exact solution is given by

$$
u(t)= \begin{cases}e^{-\frac{2 t}{\varepsilon}}, & 0 \leq t \leq 1 \\ \frac{e^{-\lambda_{1}(t-1)}-e^{-\lambda_{2}(t-1)}}{\sqrt{1+\varepsilon}}-1+e^{-\frac{2 t}{\varepsilon}}+e^{-\frac{2(t-1)}{\varepsilon}}, & 1 \leq t \leq 2\end{cases}
$$

where

$$
\lambda_{1}=\frac{1-\sqrt{1+\varepsilon}}{\varepsilon}, \quad \lambda_{2}=\frac{1+\sqrt{1+\varepsilon}}{\varepsilon} .
$$

We define the exact error $e_{\varepsilon}^{N}$ and the computed $\varepsilon$-uniform maximum pointwise error $e^{N}$ as follows

$$
\begin{aligned}
e_{\varepsilon}^{N} & =\|y-u\|_{\infty, \bar{\omega}_{N_{0}}}, \\
e^{N} & =\max _{\varepsilon} e_{\varepsilon}^{N},
\end{aligned}
$$

where $y$ is the numerical approximation to $u$ for various of $N$ and $\varepsilon$. Parameteruniform rates of convergence are computed by

$$
p^{N}=\ln \left(e^{N} / e^{2 N}\right) / \ln 2 .
$$

The values of $\varepsilon$ and $N$ for which we solve the test problem are $\varepsilon=2^{-i}, i=0,6,12,18,24 ; N=64,128,256,512,1024$. From Table 1 we observe that the $\varepsilon$-uniform experimental rate of convergence is monotonically increasing towards one, so in agreement with the theoretical rate given by Theorem 1.

TABLE 1. Errors and rates of convergence for Example 1.

\begin{tabular}{clllll}
\hline$\varepsilon$ & $N=64$ & $N=128$ & $N=256$ & $N=512$ & $N=1024$ \\
\hline $2^{0}$ & 0.008841 & 0.004804 & 0.002539 & 0.001305 & 0.000666 \\
& 0.88 & 0.92 & 0.96 & 0.97 & \\
$2^{-6}$ & 0.008783 & 0.004839 & 0.002575 & 0.001333 & 0.000676 \\
& 0.86 & 0.91 & 0.95 & 0.98 & \\
$2^{-12}$ & 0.008783 & 0.004840 & 0.002575 & 0.001333 & 0.000676 \\
& 0.86 & 0.91 & 0.95 & 0.98 & \\
$2^{-18}$ & 0.008768 & 0.004831 & 0.002571 & 0.001331 & 0.000675 \\
& 0.86 & 0.91 & 0.95 & 0.98 & \\
$2^{-24}$ & 0.008768 & 0.004831 & 0.002571 & 0.001331 & 0.000675 \\
& 0.86 & 0.91 & 0.95 & 0.98 & \\
$e^{N}$ & 0.008841 & 0.004840 & 0.002575 & 0.001333 & 0.000676 \\
$p^{N}$ & 0.86 & 0.91 & 0.95 & 0.98 & \\
\hline
\end{tabular}


Example 2. Consider the initial-value problem

$$
\begin{aligned}
& \varepsilon u^{\prime}+u+\int_{t-1}^{t} s u(s) d s=5 t^{2}-1,0<t \leq 2 \\
& u(t)=5+t,-1 \leq t \leq 0 .
\end{aligned}
$$

For this problem the exact solution is not known. Therefore we use the doublemesh principle to estimate the errors and compute solutions, that is, we compare the computed solution with the solution on a mesh that is twice as fine. The Table 2 shows our numerical results for the second problem. We measure the accuracy in the discrete maximum norm

$$
e_{\varepsilon}^{N}=\max _{i}\left|y_{i}^{\varepsilon, N}-\tilde{y}_{i}^{\varepsilon, 2 N}\right|,
$$

where $\tilde{y}_{i}^{\varepsilon, 2 N}$ is the approximate solution of the respective method on the mesh

$$
\tilde{\omega}_{2 N}=\left\{x_{\frac{i}{2}}: i=0,1,2, \ldots, 2 N\right\}
$$

with

$$
x_{i+\frac{1}{2}}=\frac{x_{i}+x_{i+1}}{2} \text { for } i=0,1,2, \ldots, N-1 .
$$

The rates of convergence are defined as

$$
p_{\varepsilon}^{N}=\frac{\ln \left(e_{\varepsilon}^{N} / e_{\varepsilon}^{2 N}\right)}{\ln 2} .
$$

The $\varepsilon$-uniform errors $e^{N}$ are estimated from

$$
e^{N}=\max _{\varepsilon} e_{\varepsilon}^{N} .
$$

The corresponding $\varepsilon$-uniform the rates of convergence are computed using the formula

$$
p^{N}=\frac{\ln \left(e^{N} / e^{2 N}\right)}{\ln 2} \text {. }
$$


TABLE 2. Errors and rates of convergence for Example 2

\begin{tabular}{clllll}
\hline$\varepsilon$ & $N=64$ & $N=128$ & $N=256$ & $N=512$ & $N=1024$ \\
\hline $2^{0}$ & 0.033904 & 0.019338 & 0.010435 & 0.005515 & 0.002835 \\
& 0.81 & 0.89 & 0.92 & 0.96 & \\
$2^{-6}$ & 0.043189 & 0.024464 & 0.013293 & 0.006977 & 0.003562 \\
& 0.82 & 0.88 & 0.93 & 0.97 & \\
$2^{-12}$ & 0.059090 & 0.033471 & 0.018187 & 0.009612 & 0.004907 \\
& 0.82 & 0.88 & 0.92 & 0.97 & \\
$2^{-18}$ & 0.064587 & 0.036332 & 0.019470 & 0.010290 & 0.005253 \\
& 0.83 & 0.90 & 0.92 & 0.97 & \\
$2^{-24}$ & 0.066203 & 0.037500 & 0.020096 & 0.010621 & 0.005422 \\
& 0.82 & 0.90 & 0.92 & 0.97 & \\
$e^{N}$ & 0.066203 & 0.037500 & 0.020096 & 0.010621 & 0.005422 \\
$p^{N}$ & 0.82 & 0.90 & 0.92 & 0.97 & \\
\hline
\end{tabular}

\section{CONCLUSiOnS}

In this paper, we presented a finite difference scheme on the piecewise uniform mesh to solve singularly perturbed initial value problem for a linear first order Volterra integro-differential equation with delay. The difference scheme is based on the method of integral identities with the use of appropriate interpolating quadrature rules with remainder term in integral form. The emphasis is on the convergence of numerical method. It is shown that the method displays uniform convergence in respect to the perturbation parameter. We have implemented the present method on two particular problems. The numerical results show that the proposed method is first order uniformly accurate and hence can be recommended for singularly perturbed Volterra delay-integro-differential equation. The main lines for the analysis of the uniform convergence carried out here can be extended to more complicated linear differential equations as well as nonlinear differential equations.

\section{REFERENCES}

[1] G. M. Amiraliyev and F. Erdoğan, "Uniform Numerical Method for Singularly Perturbed Delay Differential Equations." Comput. Math. Appl., vol. 53, no. 8, pp. 1251-1259, 2007, doi: 10.1016/j.camwa.2006.07.009.

[2] G. M. Amiraliyev and B. Yilmaz, "Fitted Difference Method for a Singularly Perturbed Initial Value Problem." Int. J. Math. Comput., vol. 22, no. 1, pp. 1-10, 2014.

[3] A. Bellour and M. Bousselsal, "Numerical solution of delay integro-differential equations by using Taylor collocation method." Math. Meth. Appl. Sci., vol. 37, no. 10, pp. 1491-1506, 2014, doi: 10.1002/mma.2910.

[4] G. A. Bocharov and F. A. Rihan, "Numerical modelling in biosciences using delay differential equations." J. Comput. Appl. Math., vol. 125, no. 1-2, pp. 183-199, 2000, doi: 10.1016/S03770427(00)00468-4. 
[5] P. A. Farrel, A. F. Hegarty, J. J. H. Miller, E. O'Riordan, and G. I. Shishkin, Robust Computational Techniques for Boundary Layers. New York: Chapman and Hall/CRC, 2000.

[6] A. D. Gaetano and O. Arino, "Mathematical modelling of the intravenous glucose tolerance test." J. Math. Biol., vol. 40, no. 2, pp. 136-168, 2000.

[7] S. Gan, "Dissipativity of $\theta$-methods for nonlinear Volterra delay-integro-differential equations." J. Comput. Appl. Math., vol. 206, no. 2, pp. 898-907, 2007, doi: 10.1016/j.cam.2006.08.030.

[8] D. He and L. Xu, "Integrodifferential Inequality for Stability of Singularly Perturbed Impulsive Delay Integrodifferential Equations.” J. Inequal. Appl., vol. 2009, no. ID 369185, pp. 1-11, 2009, doi: $10.1155 / 2009 / 369185$.

[9] C. Huang, "Stability of linear multistep methods for delay integro-differential equations." Comput. Math. Appl., vol. 55, no. 12, pp. 2830-2838, 2008, doi: 10.1016/j.camwa.2007.09.005.

[10] M. K. Kadalbajoo and V. Gupta, "A brief survey on numerical methods for solving singularly perturbed problems." Appl. Math. Comput., vol. 217, no. 8, pp. 3641-3716, 2010, doi: 10.1016/j.amc.2010.09.059.

[11] J. P. Kauthen, "A survey on singularly perturbed Volterra equations.” Appl. Numer. Math., vol. 24, no. 2-3, pp. 95-114, 1997, doi: 10.1016/S0168-9274(97)00014-7.

[12] A. H. Khater, A. B. Shamardan, D. K. Callebaut, and M. R. A. Sakran, "Numerical solutions of integral and integro-differential equations using Legendre polynomials." Numer. Algor, vol. 46, no. 3, pp. 195-218, 2007, doi: 10.1007/s11075-007-9130-2.

[13] M. Kudu, I. Amirali, and G. M. Amiraliyev, "A finite-difference method for a singularly perturbed delay integro-differential equation.” J. Comput. Appl. Math., vol. 308, pp. 379-390, 2016, doi: 10.1016/j.cam.2016.06.018.

[14] S. Marino, E. Beretta, and D. E. Kirschner, "The role of delays in innate and adaptive immunity to intracellular bacterial infection." Math. Biosci. Eng., vol. 4, no. 2, pp. 261-288, 2007, doi: 10.3934/mbe.2007.4.261.

[15] H. K. Mishra and S. Saini, "Various Numerical Methods for Singularly Perturbed Boundary Value Problems." American Journal of Applied Mathematics and Statistics, vol. 2, no. 3, pp. 129-142, 2014, doi: 10.12691/ajams-2-3-7.

[16] A. H. Nayfeh, Introduction to Perturbation Techniques. New York: Wiley, 1993.

[17] R. E. O'Malley, Singular Perturbation Methods for Ordinary Differential Equations. New York: Springer-Verlag, 1991.

[18] J. I. Ramos, "Exponential techniques and implicit Runge-Kutta methods for singularly-perturbed Volterra integro-differential equations." Neural Parallel Sci. Comput., vol. 16, no. 3, pp. 387-404, 2008.

[19] H. G. Roos, M. Stynes, and L. Tobiska, Numerical Methods for Singularly Perturbed Differential Equations. Berlin: Springer-Verlag, 1996.

[20] S. Şevgin, "Numerical solution of a singularly perturbed Volterra integro-differential equation." Adv. Difference Equ., vol. 2014, no. 171, pp. 1-15, 2014, doi: 10.1186/1687-1847-2014-171.

[21] M. Shakourifar and W. Enright, "Superconvergent interpolants for collocation methods applied to Volterra integro-differential equations with delay." Bit Numer. Math., vol. 52, no. 3, pp. 725-740, 2012, doi: 10.1007/s10543-012-0373-5.

[22] V. Subburayan and N. Ramanujam, "An Initial Value Technique for Singularly Perturbed Convection-Diffusion Problems with a Negative Shift.” J. Optim. Theory Appl., vol. 158, no. 1, pp. 234-250, 2013, doi: 10.1007/s10957-012-0200-9.

[23] S. Wu and S. Gan, "Errors of linear multistep methods for singularly perturbed Volterra delayintegro-differential equations." Math. Comput. Simul., vol. 79, no. 10, pp. 3148-3159, 2009, doi: 10.1016/j.matcom.2009.03.006. 
[24] C. Zhang and S. Vandewalle, "Stability analysis of Volterra delay-integro-differential equations and their backward differentiation time discretization." J. Comput. Appl. Math., vol. 164-165, pp. 797-814, 2004, doi: 10.1016/j.cam.2003.09.013.

[25] J. Zhao, Y. Cao, and Y. Xu, "Sinc numerical solution for pantograph Volterra delay-integrodifferential equation." Int. J. Comput. Math., vol. 94, no. 5, pp. 853-865, 2017, doi: 10.1080/00207160.2016.1149577.

\section{Authors' addresses}

\section{Gabil M. Amiraliyev}

Erzincan University, Faculty of Arts and Sciences, Department of Mathematics, 24100, Erzincan, Turkey

E-mail address: gabilamirali@yahoo.com

\section{Ömer Yapman}

Erzincan University, Faculty of Arts and Sciences, Department of Mathematics, 24100, Erzincan, Turkey

E-mail address: yapmanomer@gmail.com 